\title{
A 2020 vision for vaccines against HIV, tuberculosis and malaria
}

\author{
Rino Rappuoli ${ }^{1} \&$ Alan Aderem ${ }^{2}$
}

Acquired immune deficiency syndrome (AIDS), malaria and tuberculosis collectively cause more than five million deaths per year, but have nonetheless eluded conventional vaccine development; for this reason they represent one of the major global public health challenges as we enter the second decade of the twenty-first century. Recent trials have provided evidence that it is possible to develop vaccines that can prevent infection by human immunodeficiency virus (HIV) and malaria. Furthermore, advances in vaccinology, including novel adjuvants, prime-boost regimes and strategies for intracellular antigen presentation, have led to progress in developing a vaccine against tuberculosis. Here we discuss these advances and suggest that new tools such as systems biology and structure-based antigen design will lead to a deeper understanding of mechanisms of protection which, in turn, will lead to rational vaccine development. We also argue that new and innovative approaches to clinical trials will accelerate the availability of these vaccines.

A cquired immune deficiency syndrome (AIDS), malaria and tuberculosis are three of the most challenging infectious diseases still affecting humans (see Box 1). Since the beginning of the pandemic, AIDS has caused more than 25 million deaths, and today there are 33 million people living with HIV, 2.6 million new cases per year and 1.8 million deaths per year ${ }^{1,2}$. There are 225 million cases of malaria per year causing nearly one million deaths ${ }^{3}$. In addition, approximately onethird of the human population is infected by Mycobacterium tuberculosis, with 9.6 million new cases and 1.7 million deaths per year, and the bacterium becoming increasingly resistant to antibiotic therapy ${ }^{4}$. As one of its millennium development goals, The United Nations (UN) has elected to control and reverse the spread of these diseases by 2015 . To achieve this goal the UN is relying mostly on the expanded use of therapy, education and classical measures. These include condoms in the case of AIDS and bed nets to prevent malaria ${ }^{5,6}$.

Vaccination, which is usually the most effective intervention to control infectious diseases, was not included in the UN plan for 2015 because no vaccines are expected to be available within this period. However, new conceptual and technological advances indicate that it will be possible to develop vaccines against these diseases within the next 10 years. These advances include new prime-boost immunization regimes, new adjuvants, as well as novel methods of antigen presentation. Moreover, success will be largely dependent on our ability to use novel approaches such as systems biology to analyse data sets generated during proof-of-concept trials, leading to new insights such as the identification of correlates of protection or signatures of immunogenicity and the acceleration of largescale clinical trials. Innovative clinical and regulatory approaches will further enhance these trials.

\section{Systems biology and structure-based antigen design}

The practice of systems biology requires capturing and integrating global sets of biological data from as many hierarchical levels as possible to visualize 'emergent properties' that are not demonstrated by their individual parts and cannot be predicted from the parts alone ${ }^{7}$. The response of an individual to vaccination depends on a multitude of interacting genetic, molecular and environmental factors spanning numerous temporal and spatial scales. For this reason, the tools of systems biology are particularly well suited for the analysis of vaccine studies. These data sets include molecular measurements such as DNA sequences, RNA and protein expression levels, microRNAs, protein-protein and proteinDNA interactions and metabolite biology ${ }^{7,8}$. These measurements are made across an array of subcellular, cellular and tissue compartments including blood, immune tissues and cellular subsets derived from them. Other relevant data include genetic variation in the populations of both people and pathogens. Finally, we need to anchor the vast array of measurements in the immune phenotypes of individuals and populations. For this reason, computation is an essential element of the systems biology approach. The inference of immunological phenotypes from global data sets spanning temporal and spatial scales exceeds the capabilities of the human mind. Computational analysis transforms thousands of data points into graphical representations that will facilitate the development of detailed computational models that directly link system phenotype to the behaviour of the protein and gene regulatory networks. Once the model is sufficiently accurate and detailed, it will allow us to predict whether novel vaccines will lead to protective responses.

One example of this approach is the systems analysis of the yellow fever vaccine YF-17D, one of the most efficacious vaccines ever developed. In this case systems biology has provided insight into its mechanism of action ${ }^{9}$ and identified correlates of immunogenicity ${ }^{10}$. Expression analysis of peripheral blood mononuclear cells (PBMCs) obtained over the first 2 weeks after vaccination identified genes with expression responses or 'signatures' that are predictive of high vaccineinduced antibody and T-cell responses. The fact that these signatures were measured in peripheral blood suggests that local immune responses at the site of vaccination, which critically determine the evolution of the adaptive immunity, are reflected in this easily accessible compartment. One limitation of the study is that it could not identify a signature of efficacy because in the model used protection could not be tested. Signatures of the immune response to vaccines have further potential. For example, they can be used to predict the safety or side effects of a vaccine which would be useful in cases where the consequences of vaccination are unexpected or detrimental.

In addition to systems biology, structure-based design of novel antigens (structural vaccinology) is a powerful new tool to produce novel antigens designed to induce optimal and broadly protective immune responses ${ }^{11}$. Antigen design can improve vaccines by stabilizing the structure of

${ }^{1}$ Novartis Vaccines and Diagnostics, 53100 Siena, Italy. ${ }^{2}$ Seattle Biomedical Research Institute, Seattle, Washington 98109, USA 
difficult antigens, by exposing and improving the immunogenicity of conserved epitopes or by engineering multiple immunodominant epitopes in one molecule to induce a broad immune response. These approaches have already been used in HIV and malaria vaccine design.

\section{AIDS}

In the early 1980s when HIV was discovered, the success of the recombinant hepatitis $B$ virus vaccine produced in yeast led to the belief that all that was needed to make a viral vaccine was a recombinant subunit of the viral envelope. Unfortunately this has not been the case with HIV, one of the most difficult and challenging viruses discovered so far. The subunit vaccines derived from the HIV envelope were developed, tested in phase I and phase II clinical studies, and in the mid 1990s were ready to enter phase III efficacy studies; however, in vitro studies demonstrated that the antibodies induced by the vaccines only neutralized the virus strain used to make the vaccine and did not neutralize divergent viruses or primary viruses isolated from patients ${ }^{12,13}$. Therefore, phase III trials were postponed. A few years later VaxGen performed an efficacy trial using a vaccine composed of a mixture of the recombinant subunits from two clade $B$ viruses adjuvanted with alum. This trial was performed in approximately 5,000 high-risk volunteers mainly comprising men who have sex with men $^{14}$. A similar study with a vaccine composed of a mixture of clade $\mathrm{B}$ and clade $\mathrm{E}$ envelopes (AIDSVAX $\mathrm{B} / \mathrm{E}$ ) was started in Thailand on approximately 2,500 drug users ${ }^{15}$. The negative results of these trials were perhaps not surprising given the great antigenic diversity of the virus and the inability of the vaccines to induce antibodies able to neutralize primary isolates. The failure of the antibody-based vaccine encouraged the scientific community to focus on T-cell-mediated immunity. It had been shown that $\mathrm{CD}^{+} \mathrm{T}$ cells against broadly conserved epitopes could be induced in nonhuman primates and that these were able to blunt the peak of viraemia during primary infection and maintain a low viral load for a long time after infection $^{12}$. The enthusiasm for T-cell-based vaccines led to the design of the STEP trial, an efficacy study involving 3,000 people who were immunized either with a non-replicating adenovirus 5 (MRKAd5 HIV-1) expressing Gag/Pol/Nef or placebo. The failure of this T-cell vaccine to prevent infection or to control viral load, as had been observed in nonhuman primates ${ }^{16}$, was disappointing, leading many in the field of HIV research to question the feasibility of an HIV vaccine ${ }^{17}$. It was therefore encouraging when the results of the RV144 trial were reported in the autumn of 2009. This trial was based on a prime-boost regime: priming with a canarypox expressing the subtype B HIV Gag, Pro and the subtype E gp120 (ALVAC-HIV) and boosting with the alum adjuvanted mix of gp120 AIDSVAX B/E. Conducted in 16,000 heterosexuals in Thailand, this trial yielded a modest $31 \%$ prevention of HIV infection ${ }^{18}$. Although some researchers question whether such a low efficacy is meaningful, for many the RV144 trial has renewed the hope of developing an HIV vaccine, and attempts are now being made to plan for a trial to confirm, and perhaps improve on, the results by organizing new efficacy trials based on prime-boost regimes. If successful, these new efforts could provide licensable vaccines within this decade. In the meantime, an expanded phase II trial based on a multiclade DNA priming and adenovirus 5 boost is also being conducted by the NIH Vaccine Research Center ${ }^{2}$.

The results of three failed and one marginally successful trial could be interpreted to mean that antibodies alone or $\mathrm{CD} 8^{+} \mathrm{T}$ cells alone are not effective, and that a combination of both antibodies and $\mathrm{T}$ cells offers marginal protection against disease. However, the immune responses underlying this protection are likely to be extraordinarily complex and only amenable to systems analysis. A comparison of the immune networks induced by various prime-boost and conventional regimes could lead to the identification of signatures of immunogenicity and possibly, in the future, of protection. Because no protective vaccines exist for HIV it is not currently possible to define correlates of protection. Thus, at the moment, we are restricted to measuring defined end points such as specific $\mathrm{CD}^{+}$and $\mathrm{CD}^{+}{ }^{+} \mathrm{T}$ cells and pathogen load, which can act as useful surrogates.
Some preliminary studies are encouraging. For instance, RNA expression profiles of whole blood before and after challenge in rhesus macaques vaccinated with replicating adenovirus type 5 expressing either HIV envelope protein, simian immunodeficiency virus (SIV) Gag, or SIV Nef, followed by an HIV gp140 boost were able to identify expression signatures that distinguish vaccinated from control animals ${ }^{19}$. In another prime-boost study carried out in macaques, systems analysis of RNA expression profiling of PBMCs and lymph nodes identified network signatures that predicted the magnitude of specific $\mathrm{CD} 4^{+}$and $\mathrm{CD} 8^{+}$ T-cell responses and were associated with decreased viral load (Fig. 1 and D. E. Zak et al., unpublished observations). More information may also be obtained by following up some of the clinical studies that have already been performed. For instance, a subset of infected people from the STEP trial was followed for 2 years. Analysis revealed some decrease in viral load in people that carry the HLA alleles B27, B57 and B58 that are associated with more protective $\mathrm{CD}^{+}{ }^{+}$responses ${ }^{20}$. An additional observation that is still not explained is that the people that had high titres of antiadenovirus antibodies and were not circumcised were found to have an increased risk of infection ${ }^{16}$. Preliminary systems analysis has demonstrated that high antibody titres are associated with decreased transcription of a number of antiviral innate immune pathways including the RIG-I pathway, the TLR pathways and the inflammasome (E. Andersen-Nissen et al., unpublished data).

In humans, where experimental infection with HIV is unethical, correlates of immunity may be revealed by comparing controller and progressor

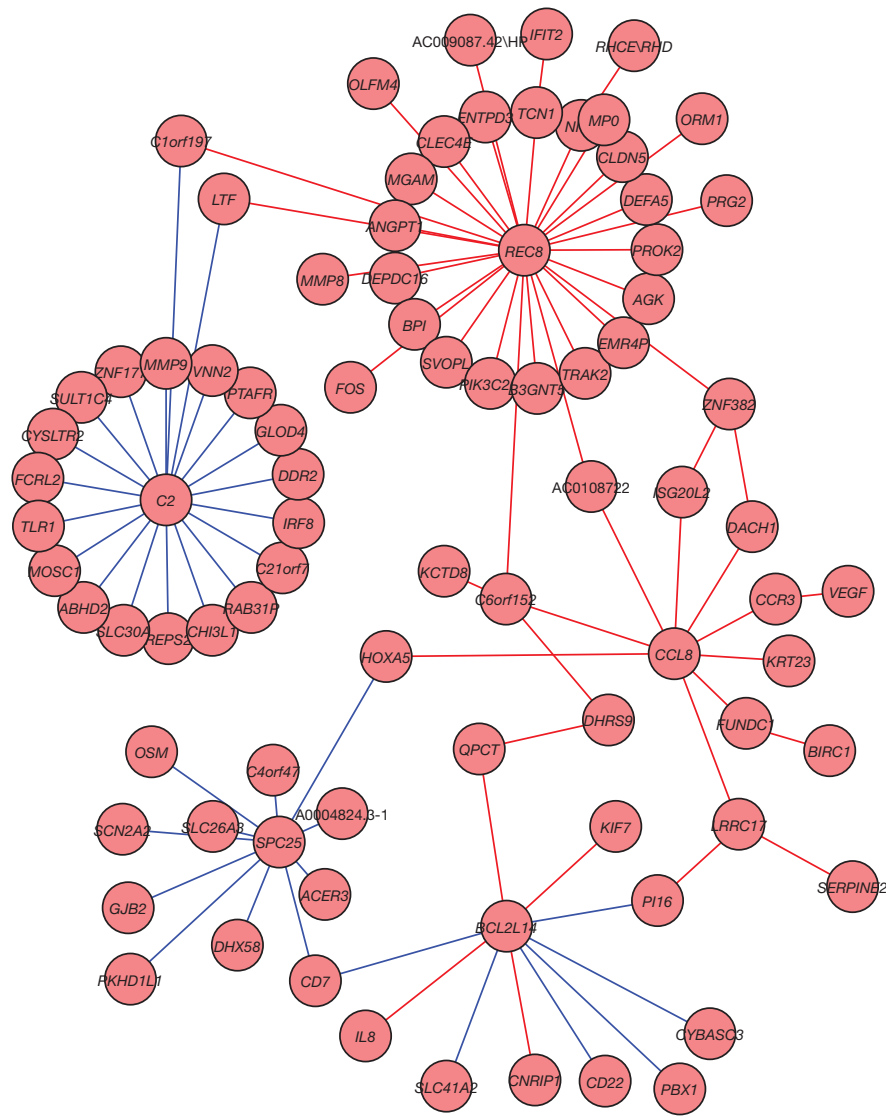

Figure $1 \mid$ Signatures that predict T-helper-cell responses after vaccination and viral load after infection. We propose that networks have stronger predictive power than do individual molecules. The network represents innate immune signatures, measured days after primary vaccination, which predict enhanced SIV Gag-specific CD4 ${ }^{+}$T-cell responses, and reduced SIV load after challenge, measured months later. In this network, the circles or 'nodes' represent genes expressed in the PBMCs of macaques 6 days after vaccination. The lines between the nodes ('edges') represent associations between them. The edges indicate which combination of genes is predictive of Gag-specific $\mathrm{CD} 4{ }^{+}$ $\mathrm{T}$ cells (blue edges) or SIV load (red edges). 
populations of infected individuals (Fig. 2). HIV controllers are HIVinfected people who control viral levels sufficiently that they never progress to AIDS. A subset of these individuals control HIV through immune mechanisms. Thus, analysis of what differs between the HIV-specific immune responses of this subset and those of HIV progressors may shed light on the particular immune responses that must be elicited in the general population for an HIV vaccine to be effective. Whereas many protective genetic variations have been identified in controller populations (particularly in the major histocompatibility complex), functional differences in their HIV-specific CD8 T cells, for example, have also been identified. Deciphering the molecular networks that control these functional differences will be useful for rational vaccine design.

In the case of HIV, structure-based antigen design has been used to engineer novel gp120 molecules that are more stable, are able to expose better the universally conserved CD4 binding site and can capture broadly neutralizing antibodies ${ }^{21}$. Similar approaches have also been used to produce influenza HA molecules that induce antibodies against the conserved regions of the haemagglutinin located in the HA2 region $^{22}$. In addition, today it is possible to use systematic approaches to map the repertoire of the human antibody response, identify the immunodominant neutralizing epitopes of different HIV variants and clades, and build the basis for engineering new envelope proteins able to provide broad protection.

\section{Tuberculosis}

In the case of Mycobacterium tuberculosis, a vaccine is available and still used to vaccinate newborns in countries with a high risk of tuberculosis infection. The vaccine was formulated a century ago and consists of Bacillus Calmette-Guerin (BCG), an attenuated strain of Mycobacterium bovis ${ }^{23,24}$. Although the overall efficacy of BCG is controversial, most agree that the vaccine is able to prevent disseminated disease and mortality in newborns and children. However, it is not able to prevent chronic infection nor to protect against pulmonary tuberculosis in adults. As a consequence, $M$. tuberculosis establishes a latent chronic infection that reactivates when there is a decrease in immune surveillance, for example in aged people, in individuals with genetic immune defects, and in those whose medication blunts their immune responses, such as a patients treated with antibodies against tumour necrosis factor- $\alpha$. Immune suppression caused by HIV has become an extremely important factor in the reactivation of tuberculosis ${ }^{25}$, and in the 15 million people co-infected by HIV and tuberculosis it is the major cause of mortality in this population ${ }^{26}$. Altogether, approximately two billion people carry a latent tuberculosis infection and approximately $10 \%$ will progress to active disease at some time. There are 12 vaccines against tuberculosis currently in clinical trials. Several of them are subunit vaccines consisting of recombinant antigens such as the Mtb72F fusion protein or the Ag85B-ESAT-6 fusion protein delivered with the adjuvant AS02, the Ag85-TB10.4 fusion protein delivered with the adjuvant IC31 (ref. 27), the

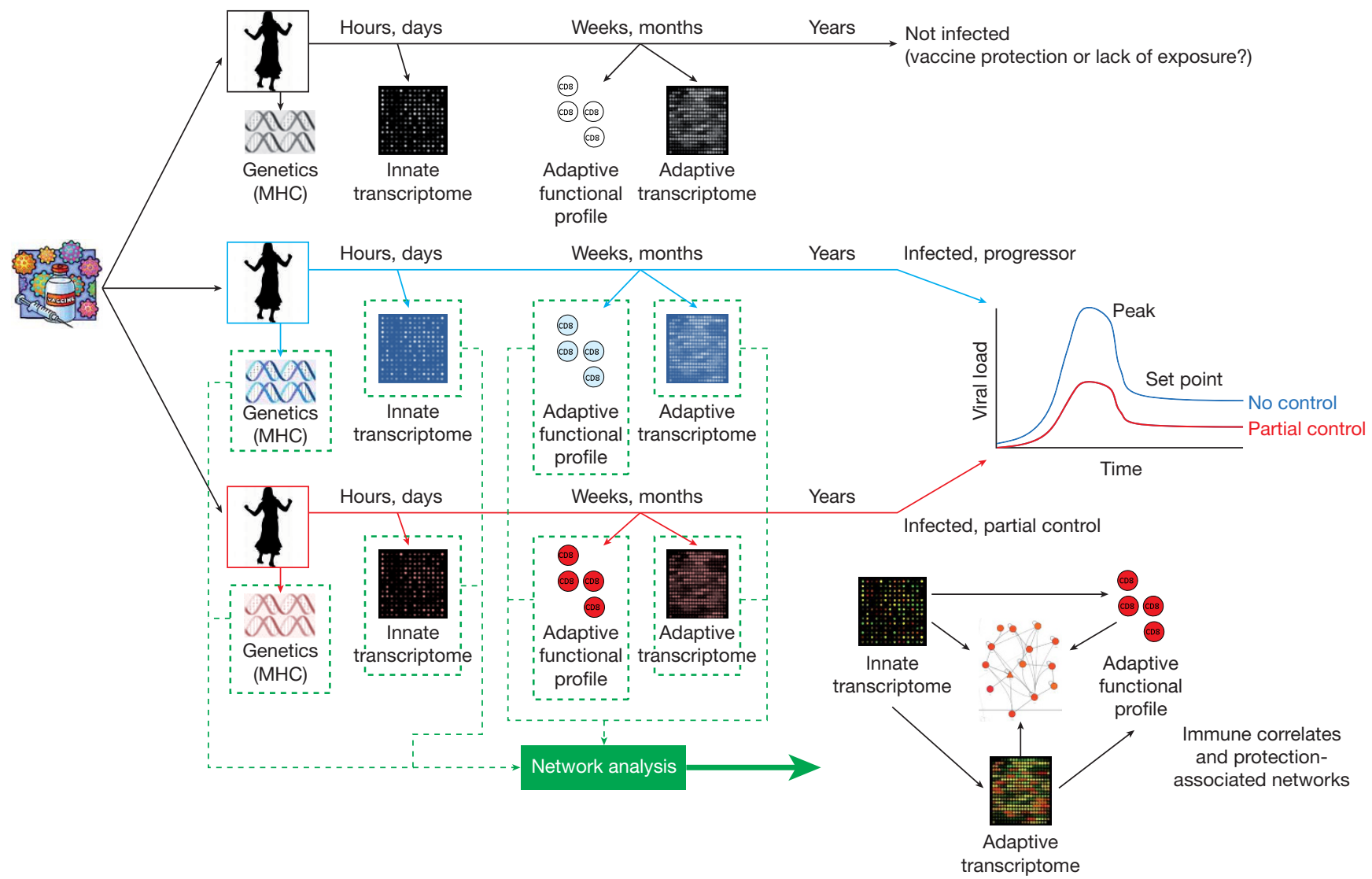

Figure $2 \mid$ Identifying novel regulatory networks associated with protection against HIV using large-scale clinical trials. Two large-scale HIV vaccine trials have shown only modest, if any, efficacy overall. Follow-up analyses are being performed to determine whether specific subgroups of participants acquire protective responses from vaccination. In principle, appropriate data mining of these trials can inform future trials by suggesting correlates of immunity and target networks that will enable rational vaccine design. For example, identification of a subpopulation of those vaccinated that became infected but controlled viral loads (red) can be compared to the population who became infected but did not control viral loads (blue). In this case, one can do systems analysis on both the innate and adaptive response to the vaccine, as well as the genetics of the individuals. Blood is collected from all of the trial participants at various times after vaccination and stored until it is known who became infected and who controlled the virus after infection. Innate cells that were collected early after vaccination and adaptive immune cells that were collected later are analysed at the molecular level using a variety of highthroughput measurements including transcriptional state, transcription-factor binding, signalling pathways, genetic variation, and metabolite and protein levels. These measurements are computationally integrated into dynamic network models that can be interpreted using visualization programs. These networks can be used as correlates of protection in subsequent vaccine trials and be used as specific molecular targets to be activated by adjuvants for rational vaccine design. This data, together with information about the individuals' major histocompatibility complex (MHC), can be used to identify better protective epitopes that can be used in future vaccines. 
fusion of Ag85B-ESAT-6-Rv2660c and a variety of antigens delivered via DNA or viral vectors ${ }^{25,28}$. Other subunit vaccines identified by reverse vaccinology have been shown to boost BCG immunity in preclinical studies $^{29}$. These subunit vaccines could be used to boost BCG vaccination in infants in the hope of preventing chronic infection. These vaccines could also be used in adolescents and adults to boost immunity induced by BCG or natural infection to delay or avoid reactivation. Another approach to improving tuberculosis vaccines is to re-engineer BCG to achieve better priming ${ }^{30}$. For example, the rBCG30 strain was engineered to overexpress antigen $85 \mathrm{~B}$ to make it more immunogenic. Indeed, in clinical trials rBCG30 was found to induce better $\mathrm{CD}^{+}$responses against Ag85B compared to wild-type BCG. Another engineered BCG strain was designed to engage the class I antigen presentation pathway based on the assumption that $\mathrm{CD}^{+} \mathrm{T}$ cells are important for protection by killing tuberculosisinfected cells; this strain was therefore engineered to express the cytolysin of Listeria monocytogenes, a protein that enables the mycobacterium to escape from the vacuole to the cytosol, where it can be presented via class I antigen presentation pathway. The vaccine strain $\mathrm{rBCGDUreC:Hly}$ also has an inactivated urease gene that allows better acidification of the vacuole and improves the release of the bacterium. Preclinical studies demonstrated that this vaccine was more attenuated and more protective than BCG; it is now being tested in phase I clinical studies.

It is interesting that after a century of tuberculosis vaccine development, and after immunizing more than 3 billion people with BCG, we still know very little about immunity to $M$. tuberculosis. We still do not know why BCG induces protection, why immunity does not prevent persistent infection, what immune response would be needed to achieve sterile immunity or to prevent reactivation of latent infection. None of these questions has been answered using conventional technologies. Progress in this field will require a more comprehensive approach, such as systems biology, to test and compare different vaccines in the field and to dissect the mechanisms associated with protection. Information about immunity to tuberculosis can also be obtained by studying infected individuals. Two recent studies ${ }^{31,32}$ used systems approaches to compare the transcripts in the blood of individuals with active infection to those of individuals who were latently infected. This investigation identified subsets of genes that correlated with the extent of the disease ${ }^{31}$. Although these signatures are not related to tuberculosis vaccine efficacy or immunogenicity, identification of the pathways associated with tuberculosis disease progression may help to define pathways that can be targeted in new vaccines.

\section{Malaria}

It has been known since 1967 that immunization with irradiated sporozoites can protect mice from infection with Plasmodium berghei ${ }^{33}$. It was subsequently found that humans immunized with the bites of $>1,000$ irradiated sporozoite-carrying mosquitoes were $100 \%$ protected from infection when challenged within 9 weeks $^{34}$. Natural infection in endemic areas also results in protection. This is why malaria causes very severe disease and mortality in infants, children and in naive adults, but causes only mild disease in adults living in endemic areas ${ }^{35,36}$. The observed immunity, however, does not last indefinitely because immune people who live abroad for a period of time become susceptible again to severe malaria when they travel back to endemic countries ${ }^{37}$.

The immunity provided by complex antigens such as irradiated sporozoites and natural infection has been very difficult to replicate using purified antigens. The best results have been obtained using the circumsporozoite protein, the most abundant antigen on the surface of the sporozoites. This protein is known to induce antibodies that inhibit the invasion of hepatocytes by sporozoites and to induce T-cell responses capable of killing infected liver cells. The antigen was expressed in a viral-like particle known as RTS, $\mathrm{S}^{38}$. The particle comprises 189 amino acids of the circumsporozoite antigen containing the repeat and terminal region fused to the 226 amino acids of the hepatitis $B$ surface antigen (RTS) and the non-fused hepatitis B surface antigen.

Because the immunogenicity of RTS,S was found to be better than any recombinant circumsporozoite antigen previously expressed, it was mixed with different adjuvants and eventually used to immunize adult volunteers that were then challenged with infected mosquito bites. Surprisingly, of the three groups immunized with the RTS,S antigen, the groups receiving vaccines adjuvanted with alum plus monophosphoryl lipid A (MPL) $(\mathrm{AS} 04)^{39}$ or with the oil in water emulsion AS03 were not protected, whereas the group receiving the vaccine adjuvanted with the oil in water emulsion plus MPL and QS21 (AS02) were 86\% protected from infection $^{40}$. Interestingly, no relevant differences in antibody titres or T-cell immunity were observed between the protected group and the nonprotected groups, indicating that the quality rather than the quantity of $\mathrm{B}$ and $\mathrm{T}$ cells was the key for protection. Unfortunately, at the time of this challenge study systems biology approaches were not yet available and the tools to evaluate the quality of the immune responses were limited, so that the development of the RTS,S vaccine continued empirically, without knowing why it had been so efficacious.

The vaccine was therefore tested in several clinical trials in adults and infants where it showed short-term efficacy in preventing infection ranging from $34 \%$ to $66 \%$, and protection of $30 \%$ against clinical malaria ${ }^{41,42}$. The vaccine was then reformulated with a different adjuvant containing liposomes plus MPL and QS21 (AS01) and tested for efficacy; it induced shortterm protection of $56 \%$ (ref. 43 ) during the first 8 months that decreased to $45 \%$ at 15 months $^{44}$. Subjects are currently being enrolled for phase III efficacy trials that are expected to provide data for registration of the vaccine for use in infants and children within the next 4 years (see http://www. gavialliance.org/resources/RTS_S_fact_sheet_Oct15_FINAL_version_3. pdf). Several other approaches to malaria vaccine development have included recombinant antigens from the merozoite and gametocyte forms of the parasite, DNA- and vector-based vaccines, and irradiated sporozoites $^{45,46}$.

In light of this limited success, a comprehensive approach based on reverse vaccinology to search the genome for the best protective antigens may be necessary to develop a multicomponent vaccine that is able to confer full and long-lasting protection. Systems biology is an ideal approach to look for non-obvious differences between protective and non-protective immunity. The availability of a well-validated human challenge model where complex vaccines based on sporozoites will induce protection only if the sporozoites are irradiated but still alive, and the availability of a simple vaccine like RTS,S that can only induce protection when combined with a particular adjuvant such as AS02, represent a unique resource to look for network signatures that may distinguish a protective immune response from a non-protective one. Systems approaches will also be critical in deconvoluting the additional complexity conferred by the heterogeneity of the parasite.

In addition, structure-based design of antigens may also help to overcome the antigenic diversity of the parasite. For instance, the apical membrane antigen (AMA-1) is one of the top vaccine candidates because it can effectively inhibit the invasion of merozoites into red blood cells. However, this antigen has a low priority in vaccines considered for advanced clinical development because its antigenic diversity compromises vaccine efficacy. However, it has been shown that chimaeras of two different antigens induce inhibition of two malaria strains ${ }^{47}$. In this case a comprehensive approach to map the immunodominant epitopes in different variants may help the design of novel molecules able to elicit a broad immune response. Additional efforts to broaden the response to AMA1 have been recently reported ${ }^{48,49}$. The ability to engineer successfully antigens able to induce broad immune responses using structurebased design of immunodominant epitopes has been shown in a recent work where the meningococcus antigen factor $\mathrm{H}$ binding protein, which is present in three different variants, was engineered to induce protective antibodies against all natural variants of the antigen ${ }^{50}$.

\section{Strengths and weaknesses of systems vaccinology Beyond signatures}

Systems biology could well enable rational vaccine design. This can be achieved when we are able to define the molecular networks that control the character and quality of specific immune responses. An integration 
of these molecular pathways with the correlates of protection will identify the specific networks within immune cells that need to be activated to achieve vaccine efficacy. These networks can then be selectively modulated by appropriately engineering antigens, adjuvants and vectors. Engineered vaccines can be optimized in an iterative process through a series of small-scale phase I clinical trials involving varied vaccine formulations that modulate the specific pathways suggested by the network analyses. These trials are smaller in scale because the capacity of the vaccine to activate specific networks associated with validated signatures predictive of immunogenicity is being tested as opposed to vaccine efficacy. Although the frequency of disease is rare, these signatures occur in most vaccinated people and therefore there is no need to involve thousands of participants to ensure a statistically significant number of cases. The different vaccine formulations can be evaluated based on the extent to which the protection-associated networks are triggered. These optimized vaccines can then be advanced to the next round of large efficacy trials. The ability to predict the efficacy of a vaccine early on in a trial will save both resources and lives.

\section{Criticisms}

Systems biology has often been criticized as being overly reliant on computation and there are those who suggest that computers will never be able to make biological sense of the mountains of data that are generated by the high-throughput technologies. Much of this criticism is predicated on a misunderstanding of the role of computers in systems biology. Computers are not expected to come up with biological insights $a b$ initio, rather they facilitate an integration of discovery science with hypothesis-driven science to yield a holistic description of a biological system. It is also difficult to evaluate properly the success or failure of computational tools in vaccinology because, until now, trials have not been designed with systems biology analysis in mind. Another criticism of computational approaches has been the fact that most of the signatures have not yielded mechanistic insights. This has not been the intentionthe goal has been for signatures that are predictive of protection which can ultimately lead to expedited vaccine trials. If these data also provide insight into the mechanism underlying protection it would be an added bonus. It has also been said that a priori analysis of the blood is naive as circulating cells do not always represent cells primarily responsible for protection. This is true, and signatures found within lymph nodes of nonhuman primates have given a deeper understanding of responses to vaccination (L. J. Picker et al., unpublished data). At a practical level, however, blood is the only accessible means to monitor the immunological response to vaccination in humans, and correlates of protection can only be established if protective efficacy is measured in the vaccine trial.

\section{Innovative trial design to accelerate development}

During the 30 years since the discovery of HIV only four efficacy trials have been performed, an average of one trial every 8 years. Two of them have shown that anti-gp120 antibodies alone do not work; one has shown that $\mathrm{T}$ cells alone do not work; and one has shown that a prime-boost regime involving B and T cells may work. Altogether, only three hypotheses have been tested. Similarly, in the case of malaria, although the field has been able to benefit from experimental human challenge models and many vaccines have been tested in phase I studies ${ }^{51}$, only two hypotheses have been tested in field efficacy trials: peptide-based vaccines and RTS,Sbased vaccines. Remarkably, no efficacy trials have been performed yet for a new preventive vaccine against tuberculosis. The sequential approach, testing one hypothesis every 8 years as we have done so far, is a procedure that we cannot afford if we want to have an impact on disease in a reasonable timeframe. Accelerated clinical development can be achieved by performing more efficacy trials and by improving their design using system biology approaches to test several hypotheses in parallel and having an adaptive design ${ }^{52-54}$ to expand the arms that are most promising (Fig. 3).

To perform more efficacy trials we need the capacity in place in those areas where diseases are prevalent as well as an adequate budget. Efficacy trials usually require budgets close to or above 100 million US dollars during the three to five years of the trial. The scientific community is often reluctant to spend this budget. However, we should keep in mind that in the case of HIV, this is less than $10 \%$ of the annual budget spent on HIV research and development. Given that the information that is obtained from a well-designed efficacy trial is of fundamental importance (even when the trial fails to show efficacy), we believe that high priority should be given to efficacy trials.

The design of efficacy trials can be improved by testing several hypotheses in parallel. For instance, several types of priming regimes paired with various boosts could be started concurrently in a large phase II study where subsets of the enrolled people are carefully monitored by systems biology approaches to test both safety and immune responses. Vaccines that elicit qualitatively similar or different immune responses can be identified, allowing more rapid discrimination of different vaccine platforms and allowing diverse concepts to be explored. The information collected during the early phases of the trial could be used to select the best arms of the trial that could be expanded to reach the statistical power to show the efficacy required for vaccine registration. Although this approach may require larger budgets during the initial phases, overall it will save money and time and will increase the probability of success. The ability to use early signatures to predict immune responses later on and therefore make early decisions on clinical trials has been recently shown to be possible. In one case, signatures in PBMCs taken 3 and 7 days

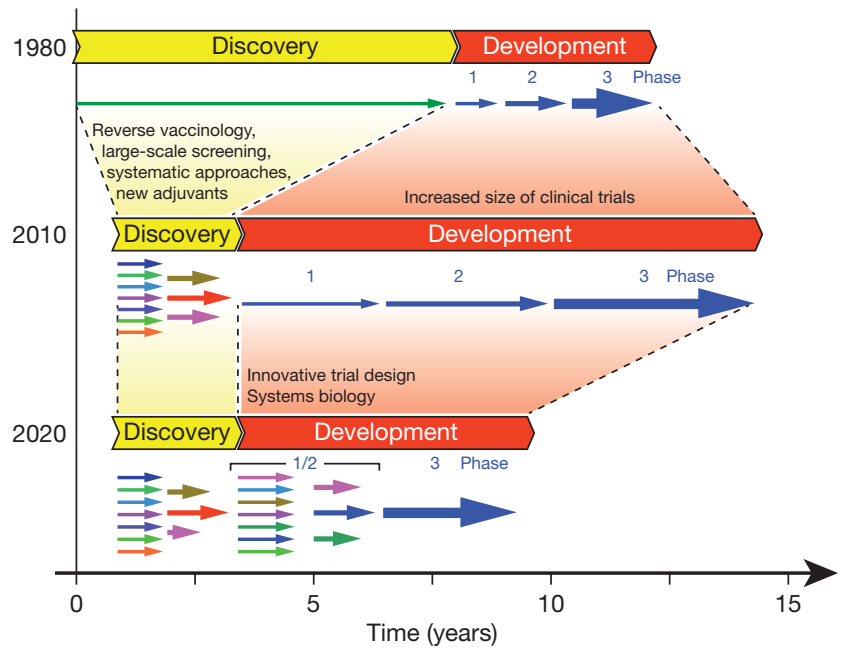

Figure $3 \mid$ Evolution of vaccine development during the last 30 years. In the 1980s discovery was the major obstacle to vaccine development. The limited technologies available allowed the development of killed, live-attenuated, toxoid, or polysaccharide vaccines only. Development used to be fast with registration requiring a few hundred subjects in clinical trials. During the 1990s, recombinant DNA technologies, conjugation and the availability of the genome sequences allowed the acceleration of the discovery phase and subsequently the discovery of vaccines against diseases that were previously impossible. High-throughput methods allowed parallel testing of multiple approaches, markedly shortening the identification of the best candidate vaccines and formulations. However, development timelines and budgets have expanded. The number of subjects required today to test safety and immunogenicity in phase I clinical trials, and in phase II studies required to define the most promising vaccine candidate, is larger than the number required to license a vaccine in 1980 . Furthermore, the number of subjects required to establish safety and efficacy in phase III field registration trials has grown beyond reasonable proportions and today the licensing of a new vaccine may require up to 80,000 people in clinical trials. If phase I, II and III studies are performed sequentially, any new vaccine starting phase I requires 10 years for clinical testing. The bottom section of the figure illustrates our expectation that systems biology and adaptive design of clinical trials will accelerate the vaccine development timelines. Multiple phase I/II clinical trials can be started in parallel and be intensively and systematically monitored by systems biology until the most promising candidate emerges. At this point the arm of the most promising candidate is expanded into a phase III registration trial, saving time compared to the sequential approach. Adapted from ref. 58. 


\section{BOX I \\ Challenging infectious diseases}

Historically successful vaccines have been developed mostly against those pathogens that can be treated by antibodies and have a stable antigen repertoire (Box 1 Figure). HIV, malaria and tuberculosis vaccines do not fall within the cluster of successful vaccines in the graph, because of antigenic variability and the requirement of T-cell immunity for protection. Developing vaccines against these pathogens requires novel approaches.

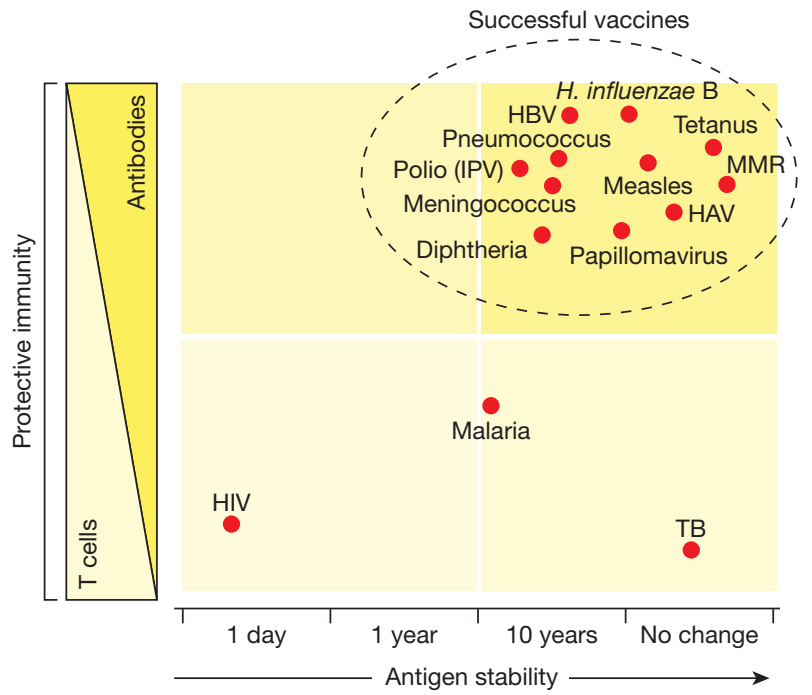

The human immunodeficiency virus (HIV) causes the acquired immune deficiency syndrome (AIDS). The genomic sequence of the virus is highly variable. The global population of HIV viruses is divided into four major clades (A, B, C and E) that are mostly present in Africa, North America and Europe, Asia, and Africa, respectively. Within each clade the sequence variability is still huge and the virus continues to evolve and mutate within each infected patient. Virus-neutralizing antibodies and T cells induced by infection or conventional vaccination elicit a narrow immune response that is not able to provide protection against all the variants of the virus.

Malaria is caused by the Plasmodium parasite that infects humans via a mosquito bite. The mosquito injects the parasite in the form of a sporozoite that rapidly migrates to the liver. After 6-7 days it is released in a different form, called a merozoite, which infects red blood cells and multiplies within them. Eventually a new form of the parasite is generated (gametocyte) that is taken up by mosquitoes again. Plasmodium falciparum and Plasmodium vivax are the main human pathogens. The different stages of the parasite have different antigenic compositions and the variability of antigens within each stage has been one of the major obstacles to vaccine development.

Tuberculosis is caused by Mycobacterium tuberculosis, a bacterium that infects human lungs where it enters and grows within macrophages. Immune cells surround the infected macrophages and form granulomas where the bacterium may become latent for a long time. Reactivation and disease can happen when the immune system weakens, a condition that is typical of HIV. Although a live-attenuated vaccine named Bacillus Calmette-Guerin (BCG), developed almost a century ago, is used in most countries, the vaccine is not able to prevent latent infection. Therefore the reactivation of tuberculosis is still a major problem and this is exacerbated by the HIV epidemic. Although there is no variability in the antigenic repertoire, protection from infection and disease is thought to be mediated mostly by T-cell immunity, and efforts to develop vaccines that are better than BCG using conventional technologies have been unsuccessful. after yellow fever vaccination were able to predict B- and T-cell responses measured at a later time $e^{9,10}$. In a second case, it was shown that that the frequency of $\mathrm{CD} 4{ }^{+} \mathrm{T}$ cells at day 21 after vaccination with avian influenza vaccine was able to predict the frequency of memory B cells, the presence of protective neutralizing antibodies, and the frequency of memory $\mathrm{CD}^{+}{ }^{+} \mathrm{T}$ cells 180 and 360 days after vaccination ${ }^{55}$.

A change in the regulatory environment could also substantially accelerate vaccine availability. When robust correlates of protection become available by classical methods or by complex systems biology approaches, they could be used to accelerate efficacy trials and, ultimately, the implementation of the vaccine. Two examples have shown the value of accelerated implementation. The first case is the meningococcus $\mathrm{C}$ vaccine. A vaccine that had been tested for safety and for its ability to induce bactericidal antibodies known to correlate with protection was introduced for mass vaccination in the United Kingdom in 1999. Within 1 year from the start of vaccination the disease had disappeared from the country with a huge impact on lives saved ${ }^{56}$. A classical approach with an efficacy trial was going to take at least another 5 years to make the vaccine available to the general population. Similar results were obtained during the meningococcus B epidemic in New Zealand. In this case, as soon as the vaccine had been shown in phase II studies to be safe and to induce bactericidal antibodies against the strain causing the epidemic, a provisional license was issued by the regulatory agency and a large-scale, countrywide immunization campaign was started. The impact of vaccination was huge and in 1 year the epidemic disappeared ${ }^{57}$, showing that when good correlates of protection are in place, a vaccine can be developed in 4 years without compromising safety.

As shown in Fig. 3, vaccine development can be accelerated by testing more vaccines and more vaccination regimens in parallel, and by an adaptive design of clinical trials that allows advancement to phase III registration trials without starting all over.

\section{Conclusions}

Marked progress has been made in the development of novel vaccines against the three most challenging infectious diseases of this century. Progress came from innovative vaccination concepts mediated by complex immunological mechanisms that we do not fully understand. Innovative design of clinical trials, testing several vaccines or vaccination regimes in parallel, and getting early information using systems biology approaches should allow the rapid testing of novel adjuvants, novel regimes of immunization and novel antigens. This should accelerate vaccine development and increase our understanding of the human immune system.

1. Joint United Nations Programme on HIV/AIDS. UNAIDS report on the global AIDS epidemic. 〈http://www.unaids.org/globalreport〉 (2010).

2. McElrath, M. J. \& Haynes, B. F. Induction of immunity to human immunodeficiency virus type-1 by vaccination. Immunity 33, 542-554 (2010).

3. World Health Organization. World Malaria Report 2010. 〈http://www.who.int/ malaria/world_malaria_report_2010/en/> (2010).

4. World Health Organization. Global Tuberculosis Control 2010. 〈http:// www.who.int/tb/publications/global_report/en/> (2010).

5. United Nations. The Millenium Development Goals Report. 〈http://www.un.org/ millenniumgoals/> (2010).

6. World Health Organization. MDG 6: combat HIV/AIDS, malaria and other diseases. 〈http://www.who.int/topics/millennium_development_goals/diseases/en/ index.html (2010).

7. Zak, D. E. \& Aderem, A. Systems biology of innate immunity. Immunol. Rev. 227, 264-282 (2009).

This is a comprehensive introduction to systems biology and how it can be used to study a complex biological property such as innate immunity.

8. Pulendran, B., Li, S. \& Nakaya, H. I. Systems vaccinology. Immunity 33, 516-529 (2010).

9. Gaucher, D. et al. Yellow fever vaccine induces integrated multilineage and polyfunctional immune responses. J. Exp. Med. 205, 3119-3131 (2008).

10. Querec, T. D. et al. Systems biology approach predicts immunogenicity of the yellow fever vaccine in humans. Nature Immunol. 10, 116-125 (2009). The work describes microarray analysis of PBMCs of subjects vaccinated with yellow fever vaccine and the discovery of signatures that correlate with $\mathrm{T}$ - and B-cell responses. 
11. Dormitzer, P. R., Ulmer, J. B. \& Rappuoli, R. Structure-based antigen design: a strategy for next generation vaccines. Trends Biotechnol. 26, 659-667 (2008).

12. Johnston, M. I. \& Fauci, A. S. An HIV vaccine-evolving concepts. N. Engl. J. Med. 356, 2073-2081 (2007).

13. Mascola, J. R. \& Montefiori, D. C. The role of antibodies in HIV vaccines. Annu. Rev. Immunol. 28, 413-444 (2010).

14. The rgp120 HIV Vaccine Study Group. Placebo-controlled phase 3 trial of a recombinant glycoprotein 120 vaccine to prevent HIV-1 infection. J. Infect. Dis. $191,654-665$ (2005)

15. Pitisuttithum, P. et al. Randomized, double-blind, placebo-controlled efficacy trial of a bivalent recombinant glycoprotein $120 \mathrm{HIV}-1$ vaccine among injection drug users in Bangkok, Thailand. J. Infect. Dis. 194, 1661-1671 (2006).

16. Buchbinder, S. P. et al. Efficacy assessment of a cell-mediated immunity HIV-1 vaccine (the Step Study): a double-blind, randomised, placebo-controlled, test-ofconcept trial. Lancet 372, 1881-1893 (2008).

17. Burton, D. R. et al. Public health. A sound rationale needed for phase III HIV-1 vaccine trials. Science 303, 316 (2004)

18. Rerks-Ngarm, S. et al. Vaccination with ALVAC and AIDSVAX to prevent HIV-1 infection in Thailand. N. Engl. J. Med. 361, 2209-2220 (2009).

A report of the results of the RV144 efficacy trial in Thailand, showing that a vaccination regime consisting of priming with a live viral vector and boosting with a recombinant protein induces a modest $31 \%$ protection from infection.

19. Palermo, R. E. etal. Genomic analysis reveals pre-and post challenge differences in a rhesus macaque AIDS vaccine trial: insights into mechanisms of vaccine efficacy. J. Virol. 85, 1099-1116 (2011).

20. Fitzgerald, D. W. et al. Step Study Protocol Team. An Ad5-vectored HIV-1 vaccine elicits cell-mediated immunity but does not affect disease progression in HIV-1infected male subjects: results from a randomized placebo-controlled trial (the Step study). J. Infect. Dis. 203, 765-772 (2011).

21. Wu, X. et al. Rational design of envelope identifies broadly neutralizing human monoclonal antibodies to HIV-1. Science 329, 856-861 (2010).

22. Wei, C.-J. et al. Induction of broadly neutralizing H1N1 influenza antibodies by vaccination. Science 329, 1060-1064 (2010).

23. Calmette, A. et al. La Vaccination Préventive contre la Tuberculose par le "BCG" (Masson, 1927).

24. Behr, M. A. et al. Comparative genomics of BCG vaccines by whole-genome DNA microarray. Science 284, 1520-1523 (1999).

25. Skeiky, Y. A. W. \& Sadoff, J. C. Advances in tuberculosis vaccine strategies. Nature Rev. Microbiol. 4, 469-476 (2006).

26. Kaufmann, S. H. Future vaccination strategies against tuberculosis: thinking outside the box. Immunity 33, 567-577 (2010).

27. Aagaard, C. etal. A multistage tuberculosis vaccine that confers efficient protection before and after exposure. Nature Med. 17, 189-194 (2011).

28. Kaufmann, S. H., Hussey, G. \& Lambert, P. H. New vaccines for tuberculosis. Lancet 375, 2110-2119 (2010).

29. Bertholet, S. et al. A defined tuberculosis vaccine candidate boosts BCG and protects against multidrug-resistant Mycobacterium tuberculosis. Sci. Transl. Med. 2, 53 ra74 (2010).

30. Horwitz, M. A., Andersen, P. A. \& Kaufmann, S. H. E. New Generation Vaccines 4th edn (Informa Healthcare, 2010).

31. Berry, M. P. et al. An interferon-inducible neutrophil-driven blood transcriptional signature in human tuberculosis. Nature 466, 973-977 (2010).

32. Maertzdorf, J. et al. Human gene expression profiles of susceptibility and resistance in tuberculosis. Genes Immun. 12, 15-22 (2011).

33. Nussenzweig, R. S., Vanderberg, J., Most, H. \& Orton, C. Protective immunity produced by the injection of $\mathrm{X}$-irradiated sporozoites of Plasmodium berghei. Nature 216, 160-162 (1967)

34. Hoffman, S. L. et al. Protection of humans against malaria by immunization with radiation-attenuated Plasmodium falciparum sporozoites. J. Infect. Dis. 185, 1155-1164 (2002).

35. Richie, T. L. \& Saul, A. Progress and challenges for malaria vaccines. Nature 415, 694-701 (2002).

36. Langhorne, J., Ndungu, F. M., Sponaas, A. M. \& Marsh, K. Immunity to malaria: more questions than answers. Nature Immunol. 9, 725-732 (2008).

37. Struik, S. S. \& Riley, E. M. Does malaria suffer from lack of memory? Immunol. Rev. 201, 268-290 (2004).

38. Gordon, D. M. et al. Safety, immunogenicity, and efficacy of a recombinantly produced Plasmodium falciparum circumsporozoite protein-hepatitis B surface antigen subunit vaccine. J. Infect. Dis. 171, 1576-1585 (1995).

39. Garçon, N., Chomez, P. \& Van Mechelen, M. GlaxoSmithKline Adjuvant Systems in vaccines: concepts, achievements and perspectives. Expert Rev. Vaccines $\mathbf{6}$, 723-739 (2007).
40. Stoute, J. A. et al. A preliminary evaluation of a recombinant circumsporozoite protein vaccine against Plasmodium falciparum malaria. N. Engl. J. Med. 336, 86-91 (1997).

This paper reports that only one of the three groups of volunteers vaccinated with the recombinant malaria antigen $\mathrm{RTS}, \mathrm{S}$ was protected from infection after malaria challenge.

41. Aponte, J. J. et al. Safety of the RTS,S/ASO2D candidate malaria vaccine in infants living in a highly endemic area of Mozambique: a double blind randomised controlled phase I/Ilb trial. Lancet 370, 1543-1551 (2007)

42. Alonso, P. L. et al. Efficacy of the RTS,S/AS02A vaccine against Plasmodium falciparum infection and disease in young African children: randomised controlled trial. Lancet 364, 1411-1420 (2004).

43. Bejon, P. et al. Efficacy of RTS,S/ASO1E vaccine against malaria in children 5 to 17 months of age. N. Engl. J. Med. 359, 2521-2532 (2008).

44. Olotu, A. etal. Efficacy of RTS,S/AS01E malaria vaccine and exploratory analysis on anti-circumsporozoite antibody titres and protection in children aged 5-17 months in Kenya and Tanzania: a randomised controlled trial. Lancet Infect. Dis. 11, 102-109 (2011).

45. Good, M. F. \& Doolan, D. L. Malaria vaccine design: immunological considerations. Immunity 33, 555-566 (2010).

46. Hoffman, S. L. et al. Development of a metabolically active, non-replicating sporozoite vaccine to prevent Plasmodium falciparum malaria. Hum. Vaccin. 6, 97-106 (2010).

47. Dutta, S. et al. Structural basis of antigenic escape of a malaria vaccine candidate. Proc. Natl Acad. Sci. USA 104, 12488-12493 (2007).

48. Kusi, K. A., Faber, B. W., Thomas, A. W. \& Remarque, E. J. Humoral immune response to mixed PfAMA1 alleles; multivalent PfAMA1 vaccines induce broad specificity. PLOS ONE 4, e8110 (2009).

49. Remarque, E. J., Faber, B. W., Kocken, C. H. \& Thomas, A. W. A diversity-covering approach to immunization with Plasmodium falciparum apical membrane antigen 1 induces broader allelic recognition and growth inhibition responses in rabbits. Infect. Immun. 76, 2660-2670 (2008).

50. Scarselli, M. et al. Rational design and structure of a meningococcal antigen inducing broad protective immunity. Sci. Transl. Med. (in press).

51. Sauerwein, R. W., Roestenberg, M. \& Moorthy, V. S. Experimental human challenge infections can accelerate clinical malaria vaccine development. Nature Rev. Immunol. 11, 57-64 (2011)

52. Freidlin, B. \& Simon, R. Adaptive signature design: an adaptive clinical trial design for generating and prospectively testing a gene expression signature for sensitive patients. Clin. Cancer Res. 11, 7872-7878 (2005).

53. Koup, R. A., Graham, B. S. \& Douek, D. C. The quest for a T cell-based immune correlate of protection against HIV: a story of trials and errors. Nature Rev. Immunol. 11, 65-70 (2011).

54. Corey, L. etal. HIV-1 vaccines and adaptive trial designs. Sci. Transl. Med. 3,79ps13 (2011).

55. Galli, G. et al. Adjuvanted H5N1 vaccine induces early $\mathrm{CD} 4^{+} \mathrm{T}$ cell response that predicts long-term persistence of protective antibody levels. Proc. Natl Acad. Sci. USA 106, 3877-3882 (2009).

This study shows that $\mathrm{CD} 4^{+}$T cells measured at day 21 after immunization with adjuvanted and normal influenza vaccine correlate with antibody levels measured 6 months and 1 year after vaccination, showing that biomarkers measured shortly after vaccination can predict long-term vaccine outcome.

56. Campbell, H., Borrow, R., Salisbury, D. \& Miller, E. Meningococcal C conjugate vaccine: the experience in England and Wales. Vaccine 27 (Suppl. 2), B20-B29 (2009).

57. O'Hallahan, J., McNicholas, A., Galloway, Y., O'Leary, E. \& Roseveare, C. Delivering a safe and effective strain-specific vaccine to control an epidemic of group B meningococcal disease. N. Z. Med. J. 122, 48-59 (2009).

58. Masignani, V., Lattanzi, M. \& Rappuoli, R. The value of vaccines. Vaccine $\mathbf{2 1}$, Suppl. 2, s100-s103 (2003).

Acknowledgements The authors wish to thank C. Mallia for editorial assistance and G. Corsi for his contribution to the artwork. The authors also would like to thank D. Zak K. Kennedy and S. Black for constructive criticism on the manuscript.

Author Information Reprints and permissions information is available at www.nature.com/reprints. The authors declare competing financial interests: details accompany the full-text HTML version of the paper at www.nature.com/nature.

Readers are welcome to comment on the online version of this article at www.nature.com/nature. Correspondence should be addressed to R.R. (rino.rappuoli@novartis.com). 